\title{
Management of asymptomatic aortic stenosis: masterly inactivity but cat-like observation
}

\author{
Julian C Vaile, Michael J Griffith
}

In patients presenting with symptoms, the natural history of significant aortic stenosis is unfavourable and the prognosis is dramatically improved by valve replacement. ${ }^{1-3}$ However, some patients remain entirely free from symptoms despite severe outflow obstruction. When these asymptomatic patients are discovered incidentally it is often assumed that their outcome will be improved by surgery. We investigated whether there was any evidence in the literature to support this supposition.

Patients with aortic stenosis are usually categorised according to haemodynamic severity of outflow obstruction. This assessment is important because of its influence on prognosis in symptomatic patients. ${ }^{4}$ It is best estimated by measuring aortic valve area, either at cardiac catheterisation by Gorlin's formula or non-invasively with echocardiography. The use of aortic valve gradients alone (either Doppler or catheter derived) is inaccurate because it ignores cardiac output. ${ }^{5}$ If a gradient is found to be borderline or low, this may result from low output and the valve area should be measured. For practical purposes and for the content of this review, "severe" aortic stenosis is indicated by a valve area of $<0.9 \mathrm{~cm}^{2}$, by a catheter derived peak gradient of $>60 \mathrm{~mm} \mathrm{Hg}$ or by a Doppler derived instantaneous gradient of $>60 \mathrm{~mm} \mathrm{Hg}$.

Acquired aortic stenosis is characterised by a long asymptomatic stage, lasting for decades. $\mathrm{Up}$ to $30 \%$ of patients with severe aortic stenosis are asymptomatic, ${ }^{4-9}$ and some patients can remain symptom free even with an aortic valve area $<0.5 \mathrm{~cm}^{2} .{ }^{10}$ In 1937 Contratto and Levine drew attention to the prognostic importance of symptoms ${ }^{11}$; they assessed severe aortic stenosis clinically, radiographically, and at necropsy and noted a short life expectancy only once the major symptoms of angina, syncope, and dyspnoea developed. Ross and Braunwald in 1968 estimated that only $3-5 \%$ of sudden deaths in acquired aortic stenosis occurred in patients without symptoms. ${ }^{1}$ The crucial issue of when (if at all) to refer asymptomatic patients for surgery requires the mortality and long term complications associated with aortic valve replacement to be weighed against their risk of death on conservative treatment.

The most current and accurate data for sur- vival after aortic valve replacement (albeit for both stenosis and regurgitation) are available from the United Kingdom Heart Valve Registry 1994 report, which quotes 30 day survival at $95 \cdot 6 \%$, one year survival at $91 \cdot 7 \%$, and five year survival at $81 \cdot 3 \%$. These figures are for all cases and refer to a clearly higher risk group than patients with asymptomatic aortic stenosis. However, they do illustrate that aortic valve replacement carries with it an acute and ongoing mortality, which is not insubstantial. The serious but non-fatal complications of prosthetic heart valves not represented by postoperative survival figures must also be considered. They include valve related thromboembolism, thrombotic occlusion of the valve, haemorrhagic complications for patients requiring anticoagulation, prosthetic valve dysfunction or paravalvar regurgitation requiring reoperation, and a higher risk of endocarditis than with a stenosed native valve. Sudden death unrelated to exercise continues to occur in these patients despite valve replacement. ${ }^{312}$ The considerable mortality and complications associated with aortic valve replacement should not be overlooked, especially in the case of an asymptomatic patient for whom prognostic benefit is the sole surgical indication.

There have been a number of reports on the outcome of conservatively managed asymptomatic patients with severe aortic stenosis. ${ }^{4-91}$ Frank et al ${ }^{13}$ confirmed that sudden death can occur in asymptomatic aortic stenosis, but their study included only three asymptomatic patients and served mainly to underline the extremely poor prognosis of severe aortic stenosis with symptoms. Chizner et $a l^{7}$ studied eight asymptomatic patients with significant isolated valvar aortic stenosis (by cardiac catheterisation) in whom an operation was not initially performed. All the patients survived (average follow up 70 months). Turina and colleagues $^{4}$ followed 17 asymptomatic patients with severe aortic stenosis (on cardiac catheterisation) - at two years no patient had died or required surgery. Kelly and co-workers $^{8}$ used continuous wave Doppler echocardiography to study 51 asymptomatic patients with significant transvalvar gradients $(50$ $130 \mathrm{~mm} \mathrm{Hg}$ ) for a mean follow up of 17 months; only two patients $(3.9 \%)$ died of cardiac cause and in both cases death was pre- 
ceded by symptoms by several weeks. In each of these studies, symptomatic patients with aortic stenosis who refused operation were followed and showed striking reductions in survival, in marked contrast with asymptomatic patients.

The largest series of patients with asymptomatic severe aortic stenosis has been reported by Pellika et al from the Mayo clinic. ${ }^{9}$ One hundred and thirteen patients with peak systolic flow velocity $>4 \mathrm{~m} / \mathrm{s}(64 \mathrm{~mm} \mathrm{Hg})$ on Doppler echocardiography who did not undergo surgery within three months were followed for a mean of 20 months. Their survival did not differ from that predicted for age and sex matched control subjects and it was better than current UK survival after aortic valve replacement. There were only three cardiac deaths presumed to be a result of the aortic stenosis; all three developed symptoms at least three months before death. All these studies reinforce the benign nature of asymptomatic aortic stenosis; in particular that the rare cardiac deaths reported were nearly always heralded by the onset of symptoms several weeks or months before.

Pellika et al also followed a group of 30 asymptomatic patients who did undergo surgery within three months ${ }^{9}$; two of these patients $(7 \%)$ died suddenly within two weeks of intervention. In some of the 30 asymptomatic patients undergoing surgery, co-morbidity was the reason given for early intervention (for example, preparation for a major non-cardiac operation), but in more than half the cardiologist believed that the severity of the stenosis alone was an indication for intervention. This provides evidence that in the 1980's at a centre of excellence (the Mayo clinic) some cardiologists recommended surgery for asymptomatic aortic stenosis on prognostic grounds alone. In the 1990 s this continues to be the practice of a significant but undetermined proportion of UK cardiologists. A possible argument in support of this practice is the need to obtain an optimal surgical result during the compensated asymptomatic phase, before irreversible myocardial deterioration occurs. This argument is based on two flawed assumptions. First, that myocardial deterioration associated with aortic stenosis is irreversible; in fact there is good evidence that ventricular dysfunction, even when advanced, is reversible after aortic valve replacement. ${ }^{312}$ Second, that most asymptomatic patients will quickly develop symptoms and thus require surgery; however, in the two largest series of initially asymptomatic patients with significant aortic stenosis, only $10 \%$ (after 17 months' follow up) and $18 \%$ (after 20 months' follow up) actually required surgery. ${ }^{89}$

We conclude that adults with isolated, haemodynamically significant aortic stenosis have an excellent prognosis without valve replacement while they remain asymptomatic. However, once symptoms develop there is no doubt that prognosis is poor and is much improved by surgery. Asymptomatic patients should not therefore undergo surgery but should be kept under careful surveillance. A probable exception is in the rare case of asymptomatic patients with progressive deterioration of left ventricular function. There is general consensus backed by a limited body of evidence that this group will fare better with an operation. ${ }^{14}$ In the remaining vast majority of asymptomatic patients, "masterly inactivity and cat-like observation" is the safest approach, such that valve replacement is performed only if symptoms develop. Of the three cardinal symptoms, dyspnoea presents latest and is associated with a shorter life expectancy than angina or syncope, although for practical purposes all three should be regarded as ominous. ${ }^{1}$

The precise nature of the careful surveillance we are recommending requires more than determining an interval between outpatient appointments. In clinical practice, asymptomatic patients are usually reviewed at long intervals (six months or more), often with an echocardiogram. While this will uncover the minority of patients with progressive deterioration of left ventricular function, it is insufficient for the prompt detection of patients in whom symptoms have recently developed. We suggest that in addition to annual follow up with echocardiography, there should be a clearly documented arrangement between the patient, the general practitioner, and cardiologist, which allows the patient to be seen in hospital within at least a week of the emergence of symptoms. As sudden death has been reported within months and even weeks of the emergence of symptoms, ${ }^{89}$ the above policy also requires that the development of symptoms is considered an urgent indication for valve replacement.

For truly asymptomatic patients with isolated, haemodynamically significant aortic stenosis there is rarely any justification for aortic valve replacement. To quote Eugene Braunwald in $1990,{ }^{15}$ in reference to the Mayo clinic paper": "these observations support a position that I have taken for many years, namely, that operative treatment is the most common cause of sudden death in asymptomatic patients with aortic stenosis."

1 Ross J Jr, Braunwald E. Aortic stenosis. Circulation 1968;37(suppl V):61-7

2 Schwarz F, Baumann P, Manthey J, Hoffman M, Schuler G, Mehmel HC, et al. The effect of aortic valve replacement on survival. Circulation 1982;66:1 105-10

3 Schwarz F, Flameng W, Langebartels F, Sesto $M$, Walter $P$, Schlepper M. Impaired left ventricular in chronic aortic valve disease: survival and function after replacement by Bjork-Shiley prosthesis. Circulation 1979;60:48-58.

4 Turina J, Hess O, Sepulcri F, Krayenbuehl HP. Spontaneous course of aortic valve disease. Eur Heart $\mathcal{f}$ 1987;8:471-83.

5 Danielsen R, Nordrehaug JE, Stangeland L, Vik-Mo $H$. Limitations in assessing the severity of aortic stenosis by Doppler gradients. Br Heart $\mathcal{f} 1988 ; 59: 551-5$.

6 Griffith MJ, Carey C, Coltart DJ, Jenkins BS, WebbPeploe MM. Inaccuracies in using aortic valve gradients alone to grade severity of aortic stenosis. Br Heart $f$ alone to grade
$1989 ; 62: 372-8$.

7 Chizner MA, Pearle DL, deLeon AC Jr. The natural history of aortic stenosis in adults. Am Heart $\mathcal{F} 1980 ; 99$ : tory of $419-24$.

8 Kelly TA, Rothbart RM, Cooper CM, Kaiser DL, Smucker ML, Gibson RS. Comparison of outcome of asymptomatic to symptomatic patients older than 20 years of age with valvular aortic stenosis. Am f Cardiol 1988;61:123-30.

9 Pellikka PA, Nishimura RA, Bailey KR, Tajik AJ. The natural history of adults with asymptomatic, hemodynamically significant aortic stenosis. F Am Coll Cardiol 1990; 15:1012-17. 
10 Lombard JT, Selzer A. Valvular aortic stenosis: clinical and haemodynamic profile of patients. Ann Intern Med 1987; 106:292-8.

11 Contratto AW, Levine SA. Aortic stenosis with special reference to angina pectoris and syncope. Ann Intern Med erence to angina pec

12 Thompson $R$ Yacoub $M$, Ahmed $M$, Seabra-Gomes $R$ Rickards A, Towers $M$. Influence of preoperative left ventricular function on results of homograft replacement of the aortic valve for aortic stenosis. Am $\mathcal{f}$ Cardiol 1979;43:929-38.

13 Frank S, Johnson A, Ross J Jr. Natural history of valvular aortic stenosis. Br Heart f 1973;35:41-6.

14 Lund $O$. Preoperative risk evaluation and stratification of long term survival after valve replacement for aortic stenosis. Circulation 1990;82:124-39.

15 Braunwald $\mathrm{E}$. On the natural history of severe aortic stenosis. $\mathcal{A}$ Am Coll Cardiol 1990;15:1018-20. 